\title{
Delay Analysis of Half Subtractor using CMOS and Pass Transistor Logic
}

\author{
Ishika Sharma \\ ME Scholar \\ NITTTR Chandigarh
}

\author{
Rajesh Mehra \\ Associate Professor \\ NITTTR Chandigarh
}

\begin{abstract}
In present day skill, designing of low power systems has emerged as one of the vital theme of electronic industries due to the point that, power consumption is drawing much of the absorption in any very large scale integration (VLSI) chip design. Design of low power circuit for high performance is the necessary main concern of VLSI technique. This paper presents designing of Half Subtractor using basic gates which are drawn by conventional CMOS and Pass Transistor Logics based on $45 \mathrm{~nm}$ technology. In comparison between the conventional CMOS half subtractor and using Pass Transistor Logic the delay is $10.5 \%$ less in PTL's half subtractor which is due to less number of transistors used in Pass Transistor Logic which in further has reduced the transistor count to $28.57 \%$.
\end{abstract}

\section{Keywords}

Half subtractor, pass transistor logic, digital circuits.

\section{INTRODUCTION}

A llow-power integration need can be defined at different design levels, such as the circuit design and the process technology level. As the modern multimedia world is

developing low power devices are in demand [1].Digital circuits which are designed by using conventional CMOS cannot fulfill these basic requirements so some solutions are found out and the outcome has resulted in the form of pass transistor logic [2]. In VLSI designing the rapid increase in demand for low power can be dealt at various logic levels, such as circuit, architectural and layout. At circuit design level substantial quantity of power can be saved by way of proper option of a logic manner. The proper option of a logic manner is value able because all significant constraints leading power dissipation, switching capacitance, transition motion, and short circuit currents are strongly prejudiced by the chosen logic style [3]. Indeed, designing high-speed low-power circuits with CMOS technology has been a main research problem. Numerous logic families have been planned and used to recover circuit routine outside that of conventional static CMOS family. In addition, due to technology growing the number of transistors on chip, the presentation of static CMOS circuits originates at considerable area where power dissipation value that may be critical, specifically for transportable appliances. Innovative devices that address the power and performance encounters must be therefore, explored [4]. There are a various logic methods through which a circuit can be realized. The proper choice of a logic strategy is significant because all important constraints governing power dissipation- switching capacitance, transition activity, and short circuit currents are powerfully prejudiced by the chosen logic design [5].

\section{HALF SUBTRACTOR}

A subtractor is combinational logic circuit which takes binary numbers as inputs and subtract one input from other input and generates their difference and borrow as outputs.

Although subtractors can be built for many binary code representations, like excess-3, gray code or even binary-coded decimal, the most public subtractors operate on binary numbers. While carrying out subtraction between the two numbers, two's complement or ones' complement is very normally used to signify the negative numbers. Also due to the affluence with which calculations are performed, many a times modifying an adder to a adder-subtraction is measured comparatively important. Other signed number representations require a more composite subtractor. Depending upon the submission of the device or upon the purpose of the application to be achieved, the inputs to the circuit device may differ from two to three. We could perhaps use an Half-Subtractor if we have two inputs though for three inputs, a Full-Subtractor can be used [6]. Basically subtractors are of two types half subtarctor and full subtractor. The logic symbol and truth table are shown below in figure 1 and logic diagram is drawn in figure 2 . The table 1 describes according ling the output produced.

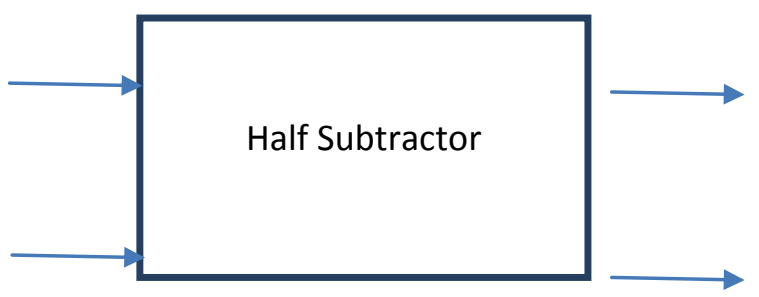

Fig. 1. 1:2 Half Subtractor Block diagram

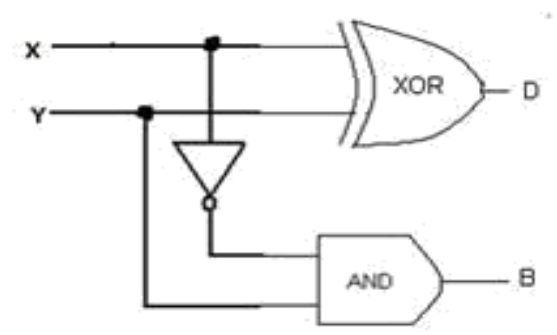

Fig 2. Logic Diagram of Conventional Half Subtractor

Table 1. Truth Table of Half Subtractor

\begin{tabular}{|c|c|c|c|}
\hline \multicolumn{2}{|c|}{ INPUTS } & \multicolumn{2}{c|}{ OUTPUTS } \\
\hline $\mathrm{X}$ & $\mathrm{Y}$ & $\mathrm{D}$ & $\mathrm{B}$ \\
\hline 0 & 0 & 0 & 0 \\
\hline 0 & 1 & 1 & 1 \\
\hline 1 & 0 & 1 & 0 \\
\hline 1 & 1 & 0 & 0 \\
\hline
\end{tabular}




\section{PASS TRANSISTOR LOGIC}

In the world of technology the insist of moveable devices are growing rapidly. Request and approval of these devices depends on the undersized silicon part, advanced speed, longer battery life and consistency [7]. Alternative technology used by logic device manufacturers for scheming integrated circuit with even few number of transistors than CMOS is Pass transistor logic (PTL) .In PTL input is feeded to both gate and source /drain stations. These circuits act as switches which use pair of NMOS and PMOS transistor called Transmission gate \& the width of PMOS is taken equal to NMOS so that both transistors can pass the signal concurrently. Figure 3 shows symbol of pass transistor logic. When $\mathrm{g}=0, \mathrm{gb}=1$ Switch is open $\&$ when $\mathrm{g}=1, \mathrm{gb}=0$ Switch is closed. So when $g=1$ : if input is ' 0 ' then output will be weak ' 0 ' and if input is ' 1 ' then output will be strong ' 1 ' [8]

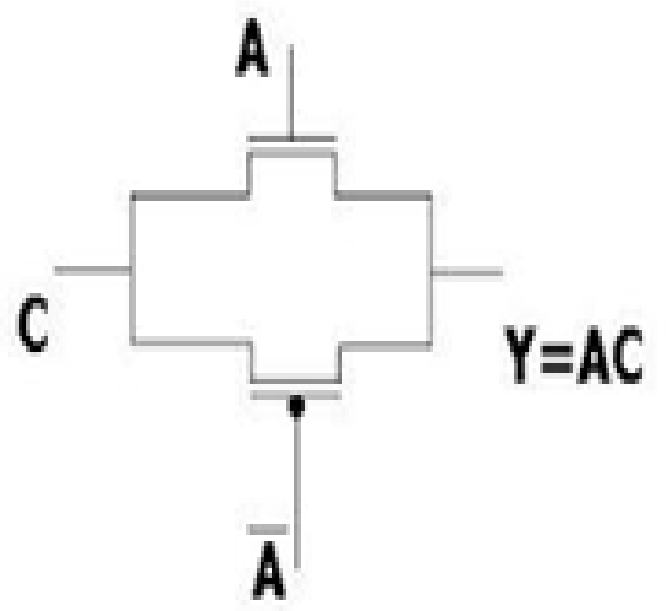

Fig. 3 Pass transistor logic

Main idea behind PTL is to use purely NMOS Pass Transistors network for logic operation. The basic difference of pass-transistor logic elegance associated to the CMOS logic style is that the source side of the logic transistor systems is linked to some input signals instead of the power lines [9]. The pass transistor is determined by a periodic clock signal and acts as an access switch to either charge up or charge down the parasitic capacitance $\mathrm{Cx}$, depending on the input signal Vin. Thus, the two possible operations when the clock signal is active $(\mathrm{CK}=1)$ are the logic "1" transfer (charging up the capacitance $\mathrm{Cx}$ to a logic-high level) and the logic "0" transfer (charging down the capacitance $\mathrm{Cx}$ to a logic-low level). In either case, the output of the depletion load NMOS inverter clearly assumes a logic low or a logic-high level, depending upon the voltage $\mathrm{Vx}$.

NMOS Pass Transistor- Logic '1' transfer:

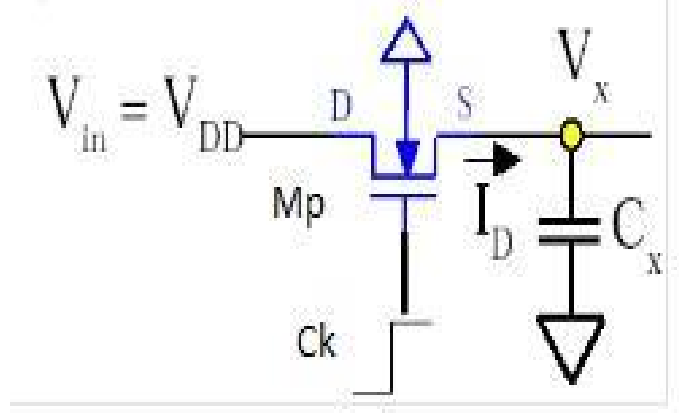

Fig. 4 NMOS Pass Transistor Logic 1 transfer.

NMOS Pass Transistor- Logic 'O' transfer:

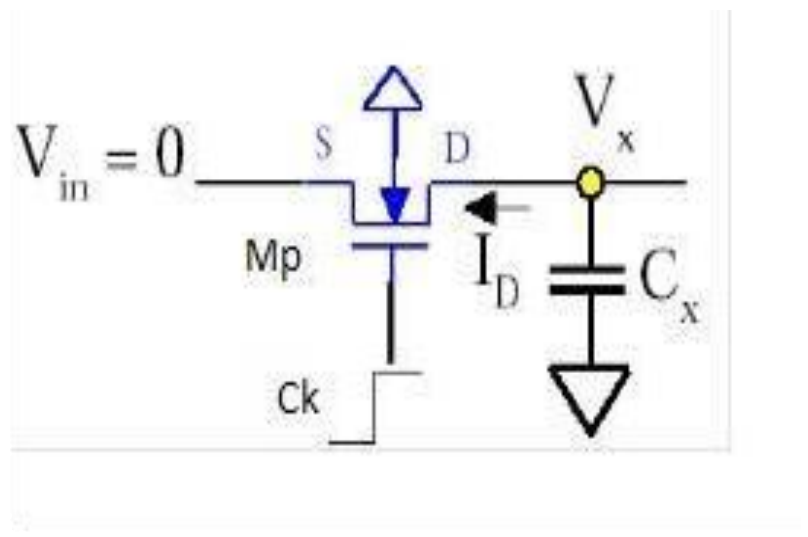

Fig. 5 NMOS Pass Transistor Logic 1 transfer.

Specifications of Pass Transistor are it is very competent in use of transistors, is potentially very resourceful layouts result, can usually be minimum size devices, propagation delays can turn out to be large in long series thread, static power dissipation is unaffected, dynamic power dissipation may be decreased. The PTL based technique uses less number of transistors compared to all other design techniques $[10,11]$

\section{SCHEMATIC DESIGNS}

The schematic design of Invertor circuit is designed using one PMOS and NMOS is designed. Similarly schematics of AND,OR, XOR GatesSimilarly the schematic of OR gate is also designed using 6 conventional transistors.

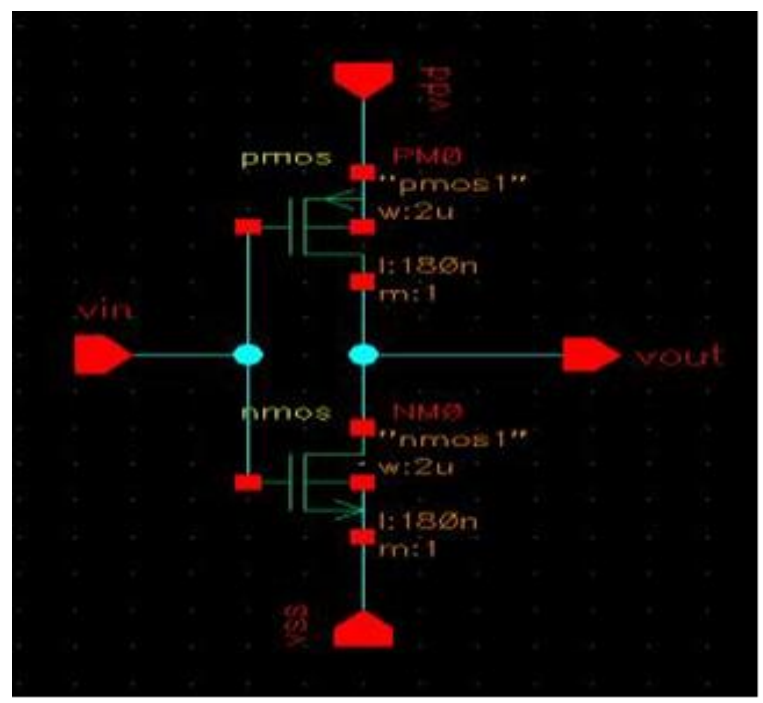

Fig. 6 Schematic of Inverter 
The schematic of AND gate is designed using 6 conventional transistors with a delay of $137 \mathrm{ps}$.

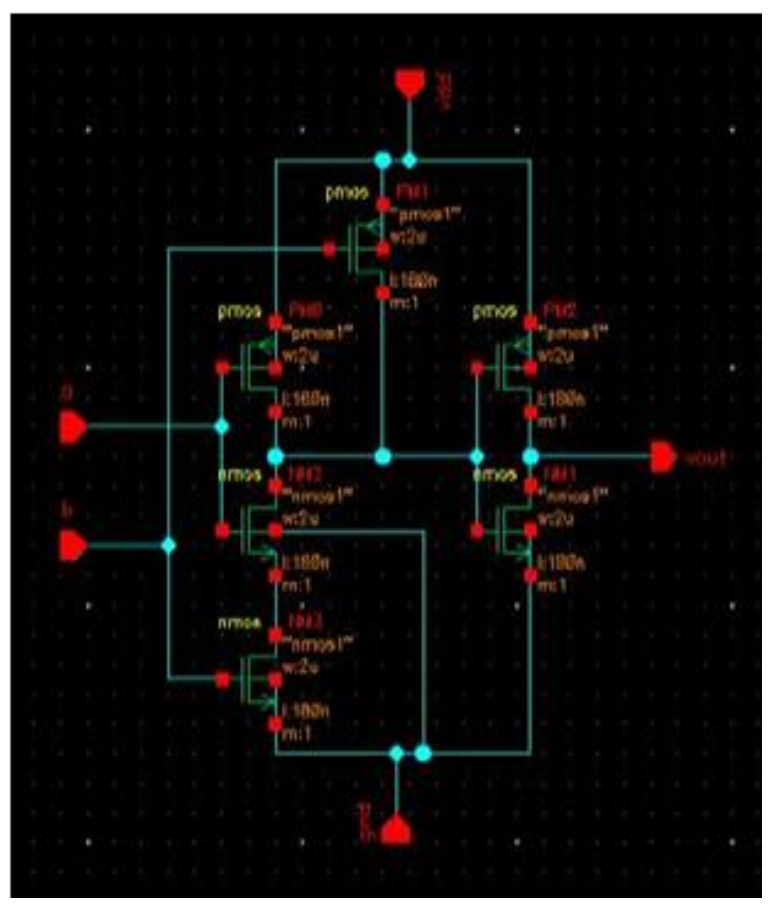

Fig. 7 Schematic of 2 Input AND Gate

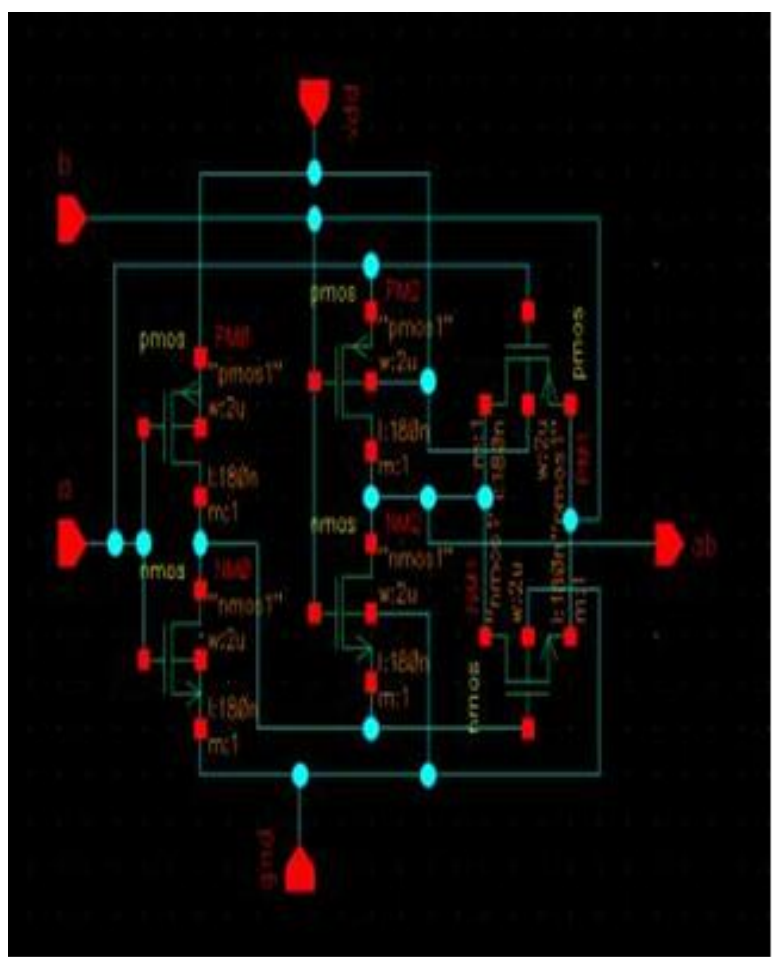

Fig. 8 Schematic of 2 Input OR Gate

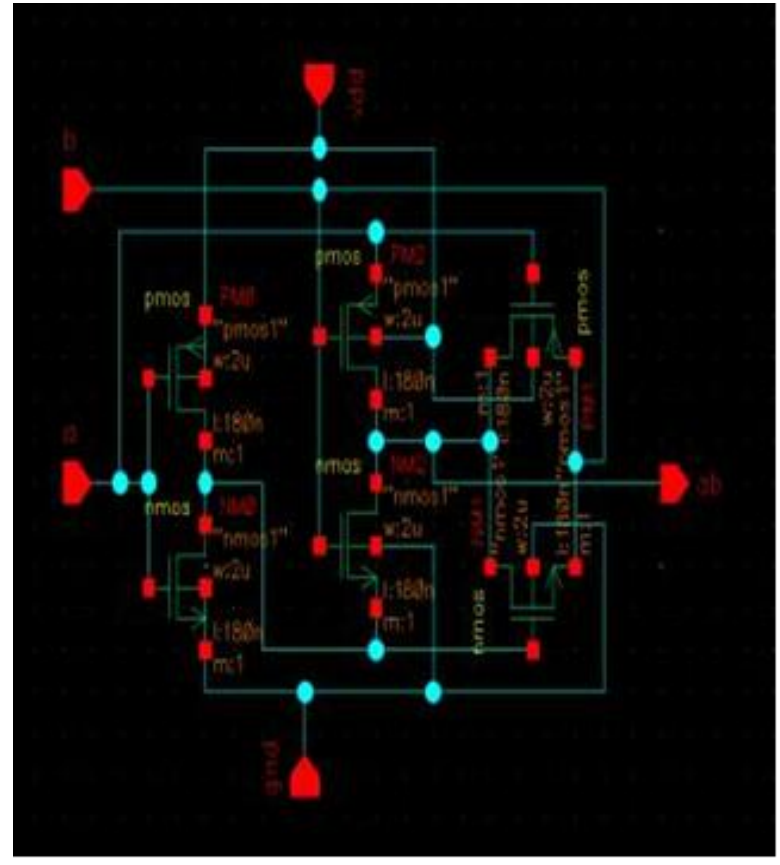

Fig. 9 Schematic of XOR gate

Schematic of XOR gate is designed using 6 conventional waveforms are generated in fig 12 . Whixh are verifying the transistors again. Truth table of half subtractor.

Now the half subtractor is designed using these basic gates and so consists of 14 conventional transistors whereas for PTL half subtractor the number of transistors used are 10.

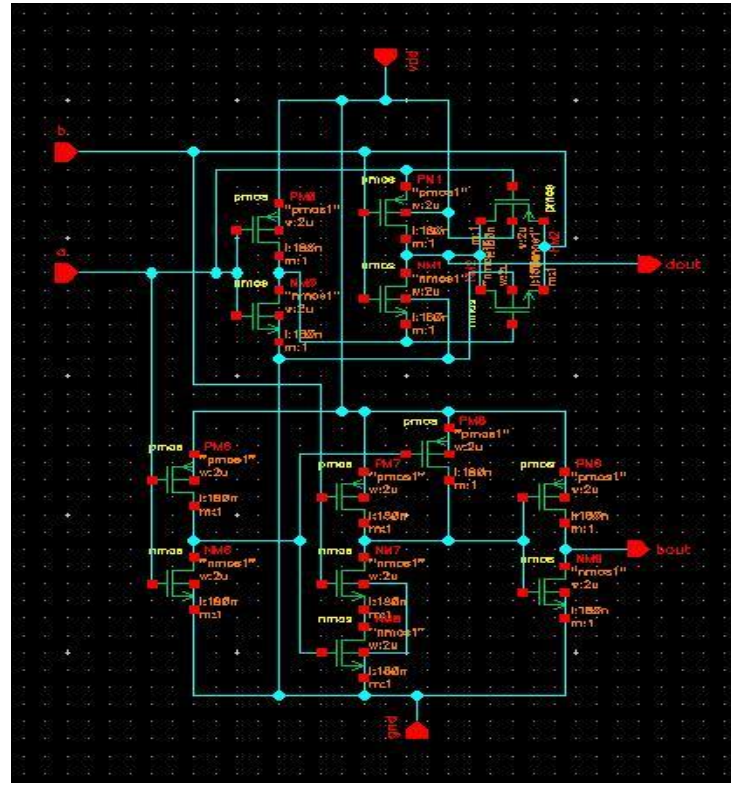

Fig. 10 Schematic of Conventional Half Subtractor 


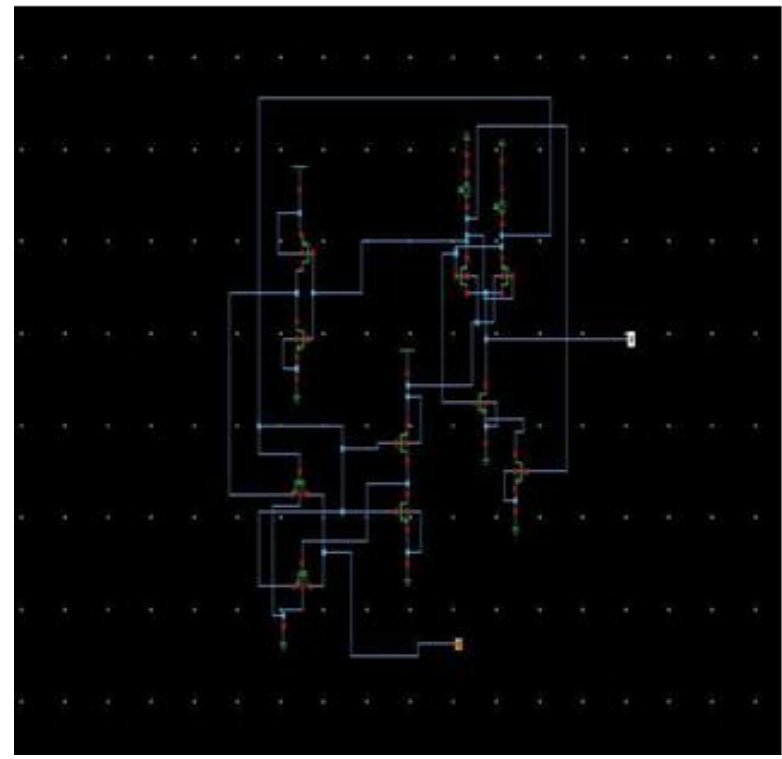

Fig. 11 Schematic of Half subtractor using PTL.

\section{RESULTS AND DISCUSSIONS}

After calculating the transient analysis of the schematics

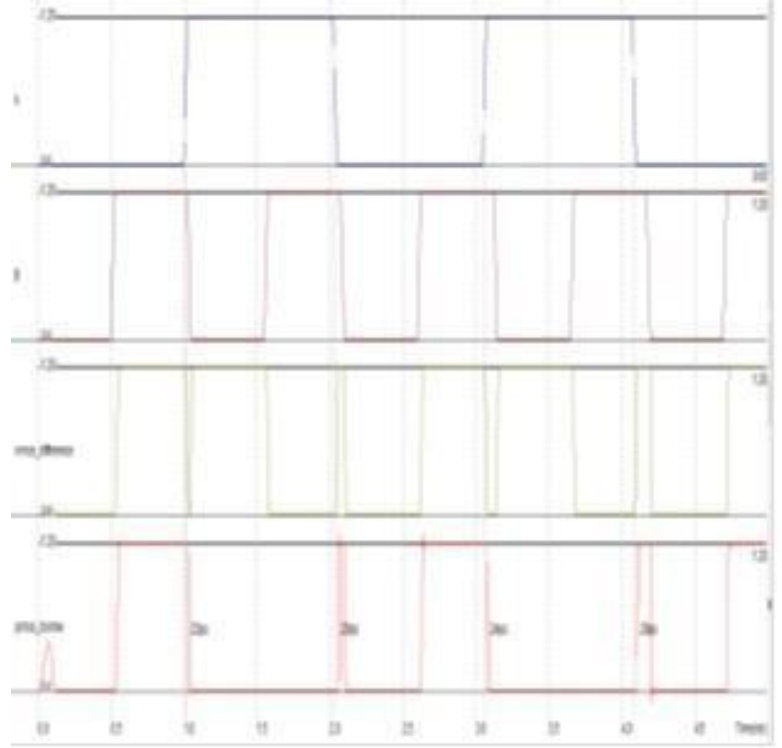

Fig. 12 Waveforms of Half Subtractor using PTL

The delay for each and every gate we have analyzed is shown in the table 2. The delay produced by half subtractor using conventional transistors is $123.5 \mathrm{p} \mathrm{sec}$ and for Pass transistor logics it is $110.5 \mathrm{p} \mathrm{sec}$ whereas the transistor count has also get reduced from 14 transistors to 10 transistors in PTL half subtractor comparative to conventional one.

Table 2. Table for delays for different gates and half subtractors

\begin{tabular}{|l|l|l|}
\hline Logic Gate & $\begin{array}{l}\text { No Of } \\
\text { Transistor }\end{array}$ & Delay \\
\hline Inverter & 2 & $137 \mathrm{ps}$ \\
\hline
\end{tabular}

\begin{tabular}{|l|l|l|}
2 input AND & 6 & $21.32 \mathrm{ps}$ \\
\hline 2 Input OR & 6 & $19.9 \mathrm{ps}$ \\
\hline 2 Input XOR & 6 & $20.1 \mathrm{ps}$ \\
\hline $\begin{array}{l}\text { Conventional } \\
\text { Half } \\
\text { Subtractor }\end{array}$ & 14 & $123.5 \mathrm{ps}$ \\
\hline $\begin{array}{l}\text { PTL Half } \\
\text { Subtractor }\end{array}$ & 10 & $110.57 \mathrm{ps}$ \\
\hline
\end{tabular}

The Bar graph below in figure 13 is for transistor count and in fig 14 delay is shown for both cases.

\section{Transistor Count}

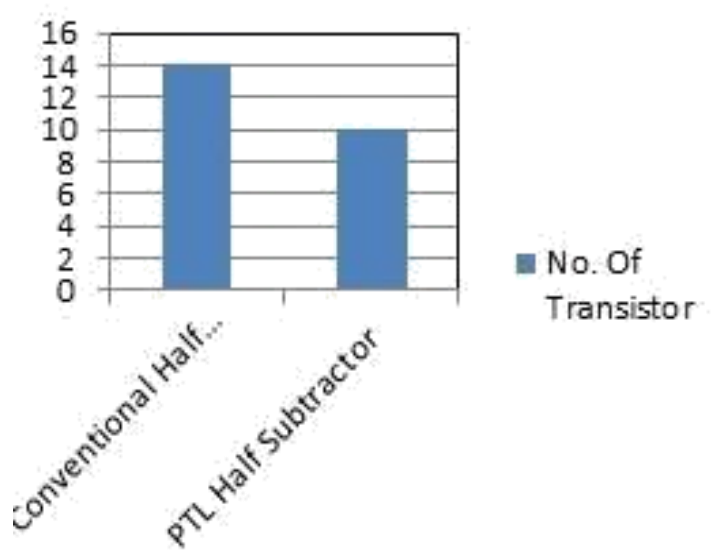

Fig. 13 Graph for Transistor count in Conventional and PTL half subtractor

\section{Delays(ps)}

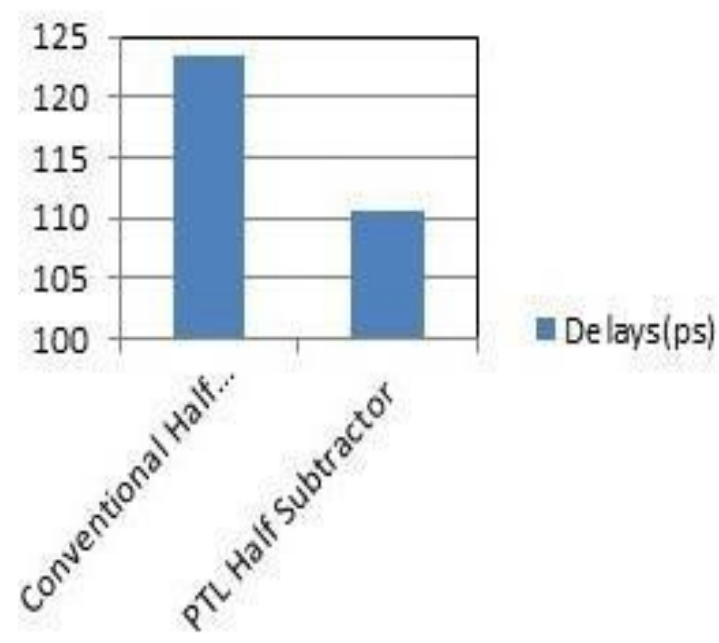

Fig. 14 Graph for delay generated by Conventional and PTL half subtractor 


\section{CONCLUSION}

From the above paper it has been concluded that the number of transistors used in designing half subtractor using PTL are less comparative to conventional CMOS as well as the delay produced by the PTL based half subtractor is $110.57 \mathrm{ps}$ which is less with respect to conventional CMOS based half subtrctor which is $123.5 \mathrm{ps}$. Not only the delay has improved but also the transistor count has reduced in pass transistor logic half subtractor as comparative to conventional half subtractors.

\section{REFERENCES}

[1] C. C. Gowda, Dr. A. R. Aswatha, "Low Power 1 Bit Full Adder Cell Using Modified Pass Transistor Logic." International Journal of Computer Science and Information Technologies, Volume 4, pp. 489-491, 2013

[2] R. K. Anand, K. Singh, P. Verma3, A. Thakur, “ Design Of Area And Power Efficient Half Adder Using Transmission Gate", International Journal of Research in Engineering and Technology, Volume: 04 Issue: 04 , pp.122-125, Apr-2015

[3] P. Sharma, A. Sharma, "Design and Analysis of Power Efficient PTL Half Subtractor Using 120nm Technology", International Journal of Computer Trends and Technology, Volume 7, No. 4, pp. 207-213, January 2015

[4] C. Khedhiri, M. Karmani , B. Hamdi, "A Differential Double Pass Transistor Logic Unit", International Journal of Computer Science Issues, Voume. 9, Issue 2, Number 1, pp. 351- 354, March 2012

[5] A. S. Narwariya, S. Akashe, "Reduction of Leakage Power in Half-Subtractor using AVL Technique based on 45nm CMOS Technology", International Journal of
Computer Applications, Volume 111, Number 1, pp. 3235

[6] T. Sood, R.Mehra, “ Design a Low Power HalfSubtractor Using $.90 \mu \mathrm{m}$ CMOS Technology", IOSR Journal of VLSI and Signal Processing, Volume 2, Issue 3 , pp.51-56, May - Jun. 2013

[7] A. Sharma, R. Mehra, " Area and Power Efficient CMOS Adder Design by Hybridizing PTL and GDI Technique" , International Journal of Computer Applications, Volume 66, No. 4, pp. 251-256, March 2013

[8] P. Singh, R. Mehra, “ Design Analysis of XOR Gates Using CMOS \& Pass Transistor Logic", International Journal of Engineering Science Invention Research \& Development, Volume 1, Issue 1, pp. 21-25, July 2014

[9] V. Choudhary, R. Mehra, " 2-Bit CMOS Comparator by Hybridizing PTL and Pseudo Logic" , International Journal of Recent Technology and Engineering, Volume 2, Issue 2, pp.29-32, May 2013

[10] A. Maheshwari, S. Luthra, "Low Power Full Adder Circuit Implementation using Transmission Gate" , International Journal of Advanced Research in Computer and Communication Engineering, Volume 4, Issue 7, July 2015, pp. 183-185

[11] Deepa, V.K. Sampath, "Analysis of Energy Efficient PTL based Full Adders using different Nanometer Technologies", IEEE Sponsered 2nd International Conference On Electronics And Communication System, 2015 